\title{
Tumor-associated fibroblasts isolated from colorectal cancer tissues exhibit increased ICAM-1 expression and affinity for monocytes
}

\author{
VERA S. SCHELLERER ${ }^{1}$, MELANIE LANGHEINRICH ${ }^{1}$, WERNER HOHENBERGER ${ }^{1}$, ROLAND S. CRONER ${ }^{1}$, \\ SUSANNE MERKEL ${ }^{1}$, TILMAN T. RAU ${ }^{2}$, MICHAEL STÜRZL ${ }^{3}$ and ELISABETH NASCHBERGER ${ }^{3}$ \\ Departments of ${ }^{1}$ Surgery, ${ }^{2}$ Pathology and ${ }^{3}$ Molecular and Experimental Surgery, \\ University Medical Center Erlangen, D-91054 Erlangen, Germany
}

Received August 30, 2013; Accepted September 27, 2013

DOI: $10.3892 /$ or.2013.2860

\begin{abstract}
Progression of colorectal cancer (CRC) is strongly associated with inflammation and other desmoplastic reactions in the tumor cell-surrounding tissue. We successfully isolated fibroblasts from the desmoplastic stroma of human CRC specimens and uninvolved colon tissue of patients treated surgically for CRC and investigated potential functional capacities. All of the isolated fibroblasts were vimentin-positive and CK-20/CD45-negative confirming the fibroblast phenotype. Differential expression patterns were detected between tumor-associated fibroblasts (TAFs) and normal tissue-associated fibroblasts (NAFs) regarding intercellular adhesion molecule-1 (ICAM-1) expression. In 11 of 12 TAF cultures, basal ICAM-1 expression was increased as compared to corresponding NAF cultures $(\mathrm{p}=0.001)$. After stimulation of the cultures with interleukin-1 $\beta, 8$ of the 12 TAF cultures presented higher ICAM-1 levels when compared with the level in the corresponding NAF cultures $(\mathrm{p}=0.001)$. Moreover, the adhesive capacity of these cultures for U937 was increased in 8 out of 10 unstimulated and in 10 out of 10 stimulated cultures when TAFs and NAFs were compared. In corresponding tumor tissue sections from the same patients, the amount of ICAM-1-positive fibroblasts was significantly higher than that in the corresponding normal colon mucosa, indicating
\end{abstract}

Correspondence to: Dr Vera S. Schellerer, Department of Surgery, University Medical Center Erlangen, Krankenhausstrasse 12, D-91054 Erlangen, Germany

E-mail: vera.schellerer@uk-erlangen.de

Abbreviations: CRC, colorectal cancer; ICAM-1, intercellular adhesion molecule-1; IL-1 $\beta$, interleukin-1 $\beta$; NAF, normal tissueassociated fibroblasts; sICAM-1, soluble intercellular adhesion molecule-1; TAF, tumor-associated fibroblasts; TNF- $\alpha$, tumor necrosis factor- $\alpha$; UICC, Union International Contre le Cancer

Key words: colorectal cancer, tumor microenvironment, intercellular adhesion molecule-1, normal tissue-associated fibroblasts, tumor-associated fibroblasts a tumor-specific effect that was maintained in the isolated cultures. These results indicate that fibroblasts from CRC tissue exhibit an increased affinity for monocytic cells. This increased intercellular interaction may contribute to elongated residence times of monocytes in CRC tissue. Therefore, these isolated fibroblasts are a useful tool for further functional investigation of desmoplastic tissue reactions in CRC.

\section{Introduction}

Colorectal cancer (CRC) is one of the leading cancers in Western countries and is responsible for $\sim 500,000$ deaths/year worldwide $(1,2)$. Inflammation is a key factor in the development and progression of CRC. The close interaction between inflammatory processes and the development of malignancy is documented by a high incidence of CRC in inflammatory bowel disease as well as a decreased CRC incidence in patients under ingestion of COX-2 inhibitors (3-5). Moreover, it is increasingly recognized that tumor initiation, growth and progression are results of complex interactions between cancer cells and the surrounding tissue, mainly consisting of fibroblasts, called the tumor microenvironment (6). Tumor-associated fibroblasts (TAFs) interact strongly with cancer cells and appear to enforce tumor progression e.g. by remodeling of the extracellular matrix, their pro-inflammatory gene signature and by guidance of cancer cells during invasion (6).

Intercellular adhesion molecule-1 (ICAM-1) is a member of the immunoglobulin superfamily and is expressed as a single cell surface glycoprotein by different cell types including fibroblasts, endothelial cells, epithelial cells, cardiomyocytes, smooth muscle cells, lymphocytes and monocytes (7-9). This molecule typically participates in cell-cell as well as cell-matrix adhesion and is also involved in cancer cell adhesion and in the immune response of tumors (9-12).

Several studies suggest that ICAM-1 plays a major role in CRC (13-17). Interestingly, the impact of ICAM-1 depends on whether the protein is expressed in a membrane-bound or a soluble form (13). Immunohistochemical staining of CRC revealed a lower incidence of lymph node and liver metastases in primary tumors expressing membrane-bound ICAM-1, whereas a lower number of ICAM-1-positive cells 
are noted in primary tumors that metastasize $(14,16,17)$. Taglia et al (16) showed that membrane-bound ICAM-1 mediates tumor cell attachment to the extracellular matrix. Thus, ICAM-1 is expected to prevent cells from detaching from the primary tumor and to attenuate metastases (16). Furthermore, membrane-bound ICAM-1 expression by cancer cells in CRC correlates with differentiation. Higher amounts of ICAM-1 were found in well-differentiated compared to lower levels in poorly differentiated tumors (16).

In contrast, the soluble form of ICAM-1 (sICAM-1) is significantly correlated with tumor stage and the development of metastasis, not only in CRC $(13,15,18-20)$ but also in other cancers such as gastric cancer $(21,22)$, breast cancer (23-25), urological malignancies (26), malignant melanoma (27), lung cancer (28), hepatocellular cancer (29) and chronic B-lymphocytic leukemia (30). At present, the source of sICAM-1 is unclear $(13,22)$ but it may be produced by proteolytic cleavage of membrane-bound ICAM-1 (31-33). Interestingly, sICAM-1 levels decrease significantly after curative surgery for CRC $(13,15)$.

In order to investigate the impact of the fibroblast phenotype on CRC progression, we isolated viable pure fibroblasts from human CRC and corresponding healthy colon mucosa of 14 patients to compare potential differences regarding their adhesive capacity mediated by ICAM-1. We demonstrated for the first time that TAFs isolated from human CRC exhibit an increased affinity for monocytic cells. Moreover, increased ICAM-1 expression was confirmed in stromal fibroblasts in CRC tissues in vivo.

\section{Materials and methods}

Tissues from the tumor and the corresponding healthy colon were obtained from 14 patients $(6$ females and 8 males; mean age: 68 years; range: $55-80$ years) undergoing elective standard surgical procedure for primarily diagnosed CRC at the Department of Surgery, University Medical Center Erlangen between February 2008 and July 2010. None of the patients received pretreatment prior to surgery. Tumors were histopathologically characterized according to the Union International Contre le Cancer (UICC). Patients with UICC stage I/II $(n=8)$ and with UICC stage III/IV CRC $(n=6)$ were included. Due to the limited life span of the isolated primary cells, not all of the tests were performed for each patient. The patient characteristics and analyses that were performed are documented in Table I. The procedure was approved by the local ethics committee, and all patients provided written informed consent.

Fibroblast isolation and cell culture. According to our published protocol (34), small pieces from the non-necrotic center of the CRC as well as from the healthy tissue at a minimum distance of $10 \mathrm{~cm}$ from the visible tumor margin were used for the generation of a single-cell suspension (34). Approximately 5 to 7 days after initial seeding, endothelial cells were removed by magnetic cell separation. The resulting negative fraction was cultivated in Dulbecco's modified Eagle's medium (DMEM) (Life Technologies, Darmstadt, Germany) supplemented with $10 \%$ fetal bovine serum (FBS) (Cambrex, Verviers, Belgium) and antibiotics (1X penicillin/streptomycin) in a $8.5 \% \mathrm{CO}_{2}$ humid atmosphere at $37^{\circ} \mathrm{C}$. Cells were grown until confluence, and were routinely tested for mycoplasma contamination. Medium replacement was performed every 3 days.

Immunocytochemistry. Cells were cultured on LabTek chamber slides (Nunc, Wiesbaden, Germany) until confluent and subsequently fixed and stained. For ICAM-1 staining, the cells were either starved for $14 \mathrm{~h}$ in DMEM-0.5\% FBS and stimulated in the same medium enriched with $200 \mathrm{U} / \mathrm{ml}$ interleukin-1 $\beta$ (IL-1 $\beta$; Roche, Mannheim, Germany) for $14 \mathrm{~h}$ or received control medium without cytokine before fixation. All slides were washed once with PBS and fixed in ice-cold ethanol for at least $20 \mathrm{~min}$. The cells were rehydrated and incubated for $2 \mathrm{~h}$ with anti-human CD45 antibody (1:10; clone T29/33), anti-human cytokeratin-20 antibody (CK-20; 1:10; both from DakoCytomation, Hamburg, Germany; clone KS 20.8) or anti-human ICAM-1 antibody (1:20; R\&D Systems, Wiesbaden-Nordenstadt, Germany; clone BBIG-I1 11C8). Subsequently, rabbit anti-mouse immunoglobulin (1:50) was added for 45 min followed by APAAP mouse (1:50; both from DakoCytomation) for another $45 \mathrm{~min}$. Staining was developed using Fuchsin chromogen (DakoCytomation). Nuclei were counterstained with Gill-III hematoxylin (Merck KGaA, Darmstadt, Germany). Slides were mounted with Aqueous Mount (Zytomed Systems, Berlin, Germany). Stained sections and cells were photographed using a Sony $3 \mathrm{CCD}$ color video camera (Sony Corporation, Munich, Germany) mounted on a Leitz Aristoplan microscope (Leica, Solms, Germany).

Stained sections were evaluated by 2 independent individuals. The ratio of ICAM-1-positive/unstained fibroblasts was evaluated for each slide, and ICAM-1 expression in TAFs and corresponding normal tissue-associated fibroblasts (NAFs) was categorized as follows: TAFs $>$ NAFs; TAFs=NAFs; $\mathrm{TAFs}<\mathrm{NAFs}$. In case of inconsistent results, a third evaluation was performed.

Adhesion assay. For the adhesion assay, 50,000 fibroblasts/well were cultivated in DMEM-10\% FBS (Life Technologies) on $1.5 \%$ gelatin-precoated LabTek 4-chamber slides (Nunc). After $6 \mathrm{~h}$, the cells were starved for $14 \mathrm{~h}$ in DMEM- $0.5 \% \mathrm{FBS}$, stimulated with or without tumor necrosis factor- $\alpha$ (TNF- $\alpha$; $1000 \mathrm{U} / \mathrm{ml}$; Roche, Grenzach, Germany) for $24 \mathrm{~h}$, and U937 cells $(500,000 /$ well) were placed in RPMI-0.5\% FBS (PAA, Cölbe, Germany) for $15 \mathrm{~min}$ at $37^{\circ} \mathrm{C}$ in a $8.5 \% \mathrm{CO}_{2}$ atmosphere. Afterwards the slides were washed with RPMI- $0.5 \%$ FBS and fixed in paraformaldehyde (Sigma-Aldrich, Munich, Germany) for at least $30 \mathrm{~min}$. Three different optical fields/chamber were documented with a Sony 3CCD color video camera mounted on a Leitz Aristoplan microscope. TAFs and corresponding NAFs with equal confluence rates were included in the final evaluation. Adherent U937 cells were counted manually, and a mean value/culture was calculated.

Immunohistochemistry. ICAM-1 staining of formalin-fixed, paraffin-embedded sections $(4 \mu \mathrm{m})$ from the tumor and normal tissues was performed as follows. Antigen retrieval was carried out using Target Retrieval Solution citrate buffer pH 6.0 (DakoCytomation). The primary rabbit anti-human ICAM-1 antibody (DakoCytomation) was diluted (1:25) 
Table I. Characteristics of the study patients and the performed analyses.

Included in analysis

UICC Vimentin/CK-20/CD45 ICAM-1 ICAM-1

Patient no. stage Gender immunocytochemistry Adhesion assay immunocytochemistry immunohistochemistry

\begin{tabular}{|c|c|c|c|c|c|c|}
\hline 1 & IV & $\mathbf{M}$ & • & & • & • \\
\hline 2 & III & $\mathrm{F}$ & • & • & • & • \\
\hline 3 & I & $\mathrm{F}$ & • & • & • & $\bullet$ \\
\hline 4 & II & M & • & & • & • \\
\hline 5 & II & $\mathbf{M}$ & • & & • & • \\
\hline 6 & III & $\mathrm{M}$ & • & • & & $\bullet$ \\
\hline 7 & III & $\mathrm{F}$ & • & • & & • \\
\hline 8 & I & $\mathbf{M}$ & • & • & • & • \\
\hline 9 & IV & $\mathbf{M}$ & • & • & $\bullet$ & • \\
\hline 10 & III & $\mathrm{F}$ & • & • & • & \\
\hline 11 & II & $\mathbf{M}$ & • & $\bullet$ & $\bullet$ & • \\
\hline 12 & I & $\mathbf{M}$ & • & • & • & • \\
\hline 13 & I & $\mathrm{F}$ & • & & • & • \\
\hline \multirow[t]{2}{*}{14} & II & $\mathrm{F}$ & • & $\bullet$ & • & $\bullet$ \\
\hline & & & $\mathrm{n}=14$ & $\mathrm{n}=10$ & $\mathrm{n}=12$ & $\mathrm{n}=13$ \\
\hline
\end{tabular}

UICC, Union International Contre le Cancer; CK-20, cytokeratin-20; ICAM-1, intercellular adhesion molecule-1. M, male; F, female.

in Antibody Diluent (Zytomed Systems) and incubated for $1 \mathrm{~h}$ in a wet chamber at room temperature. After a washing step with Tris-buffered saline (0.05 M, pH 7.6) the Rabbit Vectastain Elite ABC kit (Linaris, Dossenheim, Germany) was used as a detection system. The staining was developed using the NovaRED Substrate kit (Linaris) for $30 \mathrm{~min}$. After counterstaining of nuclei with Gill-III hematoxylin, the slides were dehydrated and mounted with VectaMount Permanent Mounting Medium (Vector Laboratories, Burlingame, CA, USA).

The stained sections were evaluated by 3 independent investigators. First, the ratio of ICAM-1-positive/negative fibroblasts (in \%) was evaluated for each slide and second, the slides of the normal and tumor tissue for each patient were compared with each other. Statistical differences were evaluated by the Student's t-test (SPSS Statistic Program, IBM, version 18.0). Stained tissue sections were photographed using an AxioCam MRc mounted on a Zeiss Imager A.2 Axio (Zeiss, Jena, Germany).

\section{Results}

TAFs and NAFs are vimentin-positive and CK-20/CD45negative. Viable fibroblasts were isolated from the surgical specimens of 14 patients and cultivated for several passages. The isolated fibroblasts presented with the typical spindleshaped morphology (Fig. 1). All cells were positive for vimentin (Fig. 1, vimentin). Contamination by epithelial and hematopoietic cells was excluded by negative CK-20 (epithelial cells) and CD45 (hematopoietic cells) staining (Fig. 1, CK-20 and CD45). Due to the limited life span of the isolated cells, ICAM-1 staining and the adhesion assays were performed with 12 (ICAM-1 staining) and 10 (adhesion assay) out of the 14 TAF/NAF pairs (Table I).

ICAM-1 expression is increased in unstimulated and stimulated TAFs when compared to ICAM-1 expression in NAFs. Immunocytochemical staining of ICAM-1 was performed in 12 out of 14 TAF/NAF pairs. In 11 out of 12 cultures, a higher number of ICAM-1-positive cells was detected in TAF when compared to the corresponding NAF cultures (Fig. 2A, compare TAF vs. NAF, unstimulated; Fig. 2B, unstimulated). After stimulation of the fibroblast cultures with interleukin- $1 \beta$ (IL-1 $\beta$ ), ICAM-1 expression was increased in all of the cultures when compared to the basal levels (Fig. 2A, compare unstimulated vs. stimulated). When comparing stimulated TAF with the corresponding stimulated NAF cultures in 8 out of the 12 cultures, TAFs presented with a higher number of ICAM-1-positive cells (Fig. 2A, compare stimulated TAF vs. NAF; Fig. 2B, IL-1 $\beta$ stimulated, $p=0.001$ ). The amount of ICAM-1-positive cells in TAFs and NAFs was equal after IL-1 $\beta$ stimulation in 4 out of the 12 cultures (Fig. $2 \mathrm{~B}$, IL-1 $\beta$ stimulated, $\mathrm{p}=0.001)$.

TAFs display a higher adhesion capacity for U937 monocytic cells when compared to NAFs. In order to estimate whether the increased ICAM-1 expression leads to increased adhesive capacity, adhesion assays using U937 monocytic cells were performed with $10 \mathrm{TAF} / \mathrm{NAF}$ pairs (Fig. 3A). In 8 out of 10 unstimulated TAF/NAF pairs, the number of adherent U937 cells/optical field was increased in the TAFs when compared to the number in the NAFs (Fig. 3B, patient no. 2, 3, 6, 7, 8, 9, 12, 14). Of note, in 3 of the pairs, the differences were statistically significant (Fig. 3B, asterisks, $\mathrm{p}<0.05)$. Comparing the average 


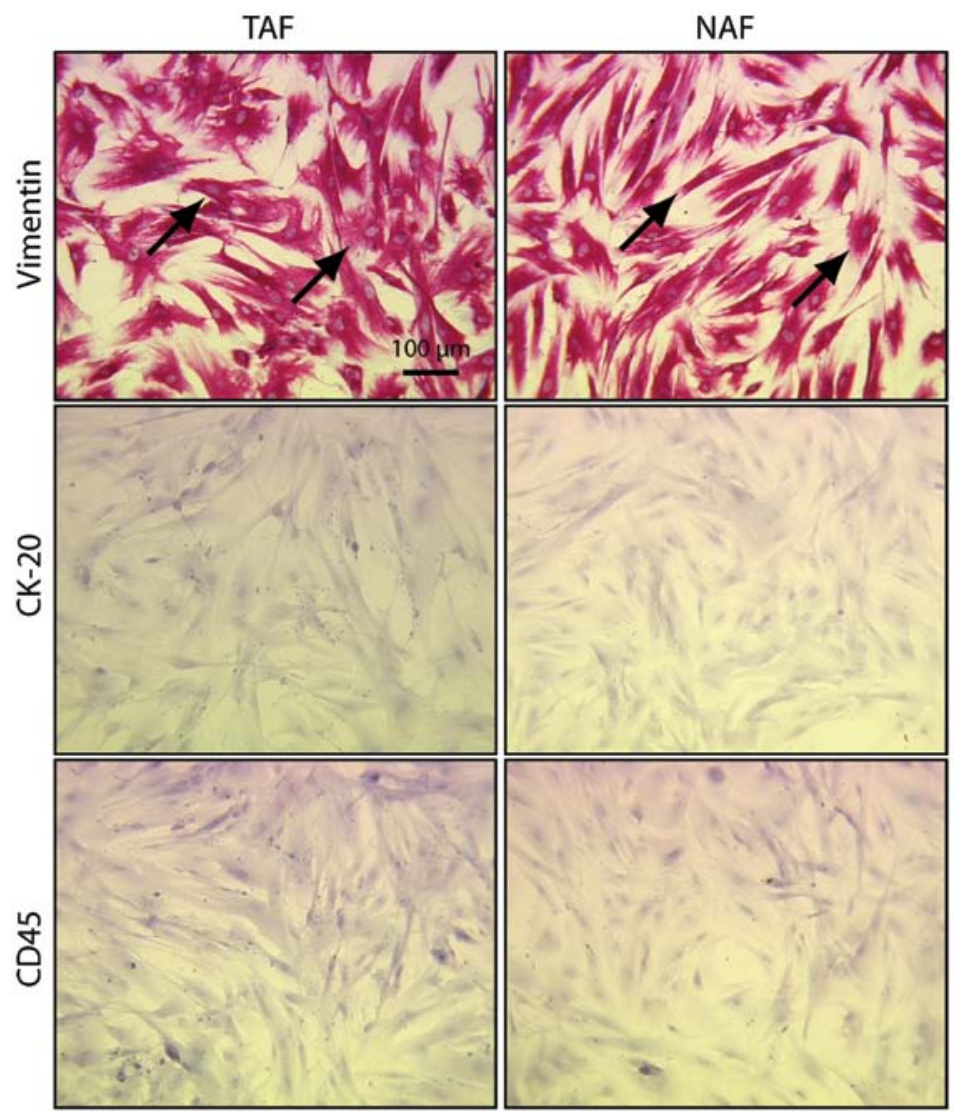

Figure 1. TAFs and NAFs isolated from CRC and healthy colon express vimentin but not CK-20 and CD45. Immunocytochemical staining of TAF and NAF cultures for vimentin, CK-20 (epithelial marker) and CD45 (hematopoietic marker). Staining was performed using the APAAP system. Counterstaining was performed with hematoxylin (blue color). Arrows indicate positive staining. Scale bar, $100 \mu \mathrm{m}$. TAFs, tumor-associated fibroblasts; NAFs, normal tissueassociated fibroblasts; CRC, colorectal cancer; CK-20, cytokeratin-20.

A
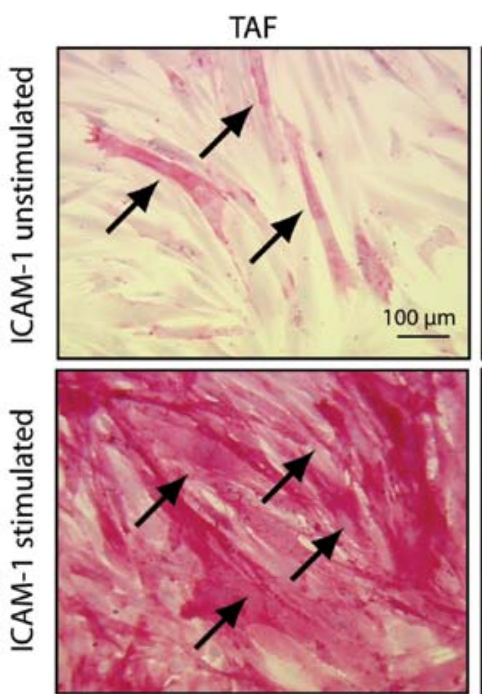
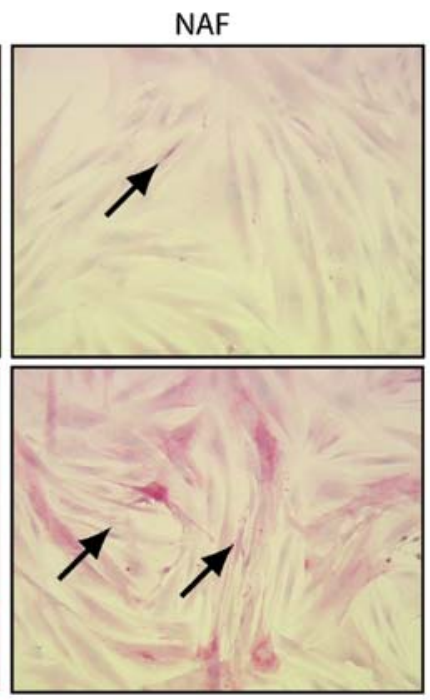

B
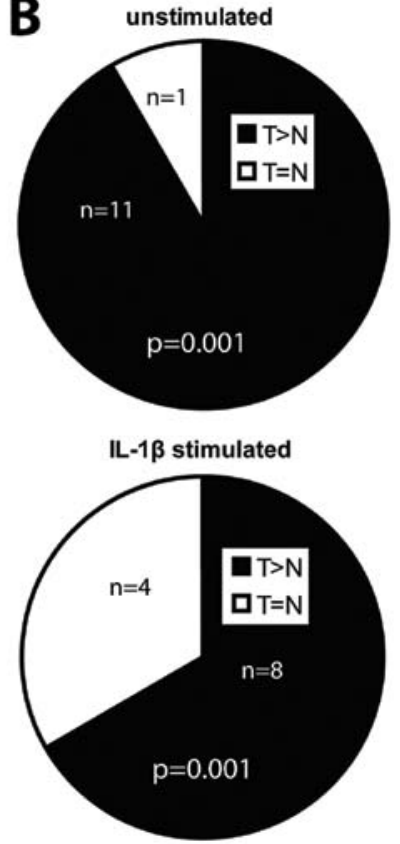

Figure 2. Unstimulated as well as stimulated (IL-1 1 ) TAFs present with a higher expression of ICAM-1 than NAFs. (A) Immunocytochemical staining for ICAM-1 in unstimulated and stimulated TAF and NAF cultures. Counterstaining was performed with hematoxylin (blue color). Arrows indicate positive staining. Scale bar, $100 \mu \mathrm{m}$. (B) Evalutation of immunocytochemical staining for ICAM-1 expression in unstimulated and IL-1 $\beta$-stimulated TAFs as compared to NAFs. The ratio of ICAM-1-positive/unstained fibroblasts was evaluated for each slide and ICAM-1 expression in TAFs and corresponding NAFs was categorized as follows: TAF $>\mathrm{NAF}$; TAF $=\mathrm{NAF}$; TAF $<\mathrm{NAF} . \chi^{2}$ test revealed significant differences between unstimulated and stimulated fibroblast cultures ( $\mathrm{p}=0.001)$. Stained sections were evaluated by 2 independent individuals. IL-1 $\beta$, interleukin-1 $\beta$; TAFs, tumor-associated fibroblasts; ICAM-1, intercellular adhesion molecule-1; NAFs, normal tissue-associated fibroblasts. 
A

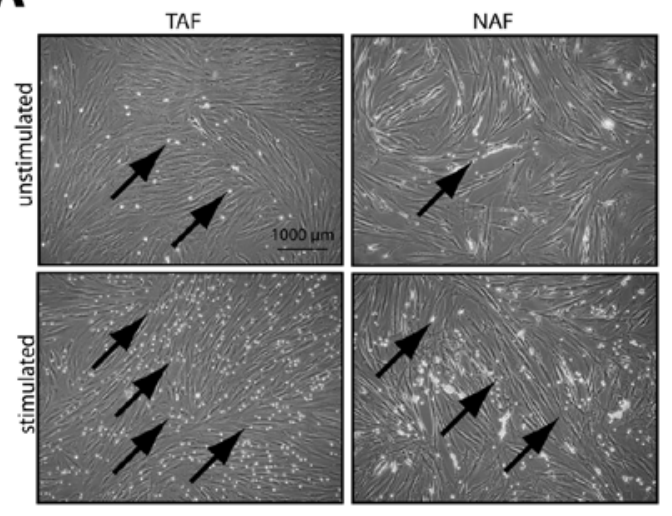

C

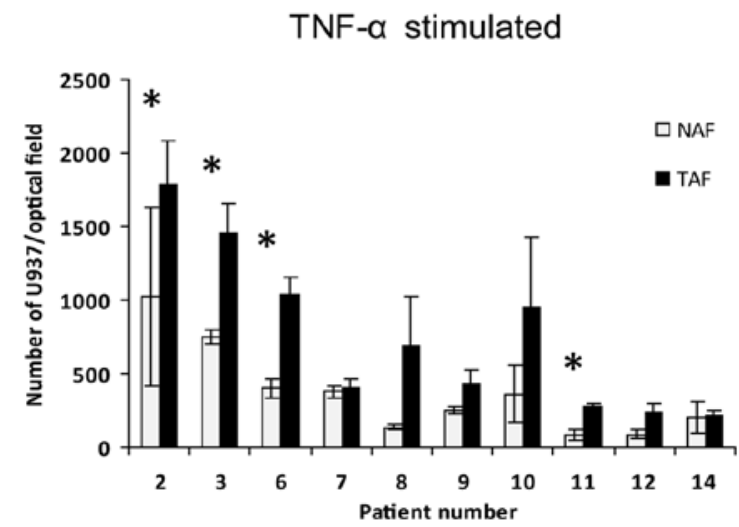

B

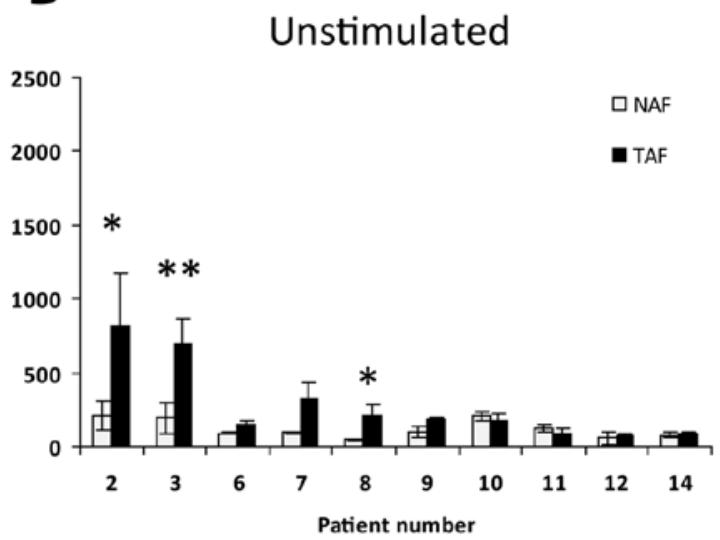

D

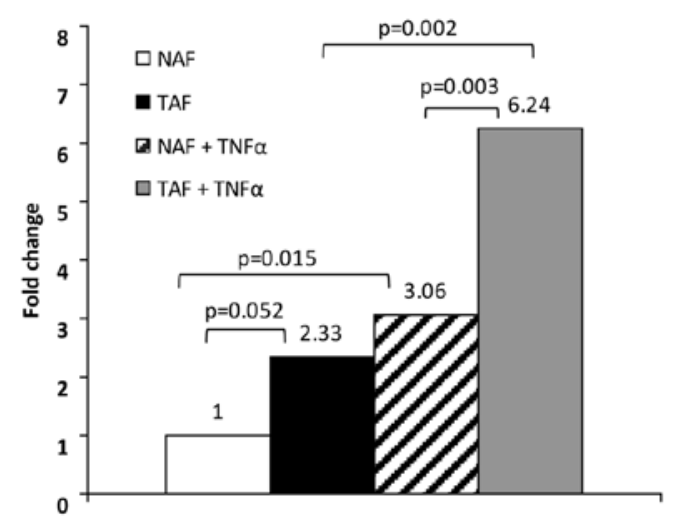

Figure 3. Unstimulated as well as stimulated (TNF- $\alpha$ ) TAF cultures showed a higher adhesion capacity for U937 when compared with the capacity of NAFs. (A) Starved fibroblast cultures (unstimulated or stimulated with TNF- $\alpha$ for $24 \mathrm{~h}$ ) were incubated with U937 monocytic cells for 15 min. After fixation, adherent U937 cells were counted manually. Arrows indicate adherent U937 monocytic cells. Scale bar, $1000 \mu \mathrm{m}$. (B) In 8 out of 10 unstimulated TAF/NAF pairs, the mean number of adherent U937 cells in 3 different optical fields was increased in TAFs when compared to the number in the NAFs (patient no. 2, 3, 6, 7, 8, 9, $12,14)$. In 3 of the pairs, the differences were statistically significant $\left({ }^{*} \mathrm{p}<0.05,{ }^{* *} \mathrm{p}<0.01\right)$. (C) After TNF- $\alpha$ stimulation in all of the 10 cultures, the adhesion capacity of TAFs was higher when compared to this capacity in the NAF cultures. In 4 of the pairs, the differences between TAFs and NAFs were statistically significant ( $\mathrm{p}<0.05)$. (D) Average fold-changes are given for unstimulated and stimulated TAFs vs. NAFs. The respective p-values are indicated in the graph (Student's t-test). TNF- $\alpha$, tumor necrosis factor- $\alpha$; TAFs, tumor-associated fibroblasts; NAFs, normal tissue-associated fibroblasts.

number of U937 cells/optical field in all unstimulated TAFs vs. NAFs, the number was increased by 2.33 -fold (Fig. 3D, compare TAF/NAF, $\mathrm{p}=0.051$ ).

After stimulation of the cultures with TNF- $\alpha$, in all of the 10 cultures, the adhesion capacity of TAFs was higher when compared to the NAF cultures (Fig. 3C). Of note, in 4 of the pairs, the differences between TAF and NAF were statistically significant (Fig. 3C, asterisks, p<0.05). The average number of U937 cells/optical field was increased in the stimulated TAFs when compared to the number in the stimulated NAFs by 2 -fold (Fig. 3D, compare TAF + TNF- $\alpha$ vs. NAF + TNF- $\alpha$, 6.24- vs. 3.06-fold, $\mathrm{p}=0.002$ ), was increased in the stimulated TAFs when compared to the number in the unstimulated TAFs by 2.67 -fold (Fig. 3D, compare 6.24- vs. 2.33-fold, p=0.002) and was also increased in the stimulated when compared to the unstimulated NAFs by 3.06-fold (Fig. 3D, compare 1 vs. $3.06, \mathrm{p}=0.015)$.

Increased ICAM-1 expression in TAFs is reflected by a corresponding increase in ICAM-1 expression in fibroblasts of the tumor tissue. In order to determine whether the increased expression of ICAM-1 in TAFs was an effect that was already present in the original tumor tissue, immunohistochemical staining was performed in 13 out of 14 tumor/normal tissue pairs (Fig. 4A). In one TAF/NAF pair, the corresponding tumor section presented only parts of an adenoma without invasive carcinoma, therefore the tumor and normal tissues of this patient were excluded from the evaluation. When counting ICAM-1-positive and -negative fibroblasts, the frequency of ICAM-1-positive fibroblasts was significantly increased in the tumor tissue as compared to the normal tissue (Fig. 4B, $\mathrm{p}<0.001)$. This indicates that the increased ICAM-1 expression of TAFs was a tumor-specific effect stably maintained in the isolated cultures.

\section{Discussion}

Recently, the understanding of fibroblast function in tumor development is gaining renewed attention. Tumor progression and the development of metastasis appear to be influenced by 
A

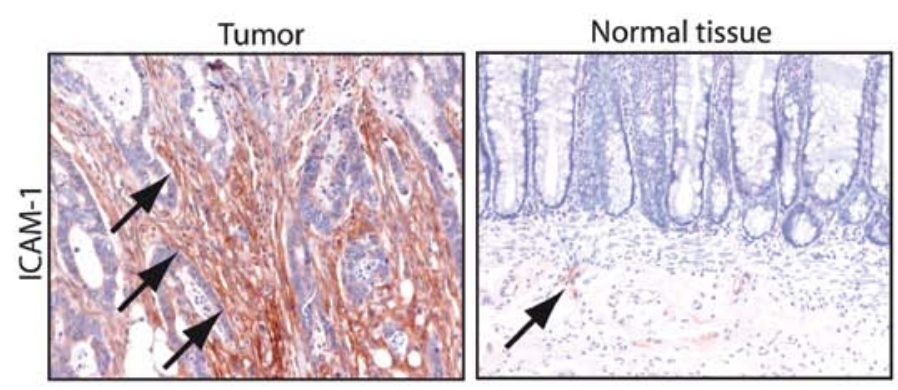

B

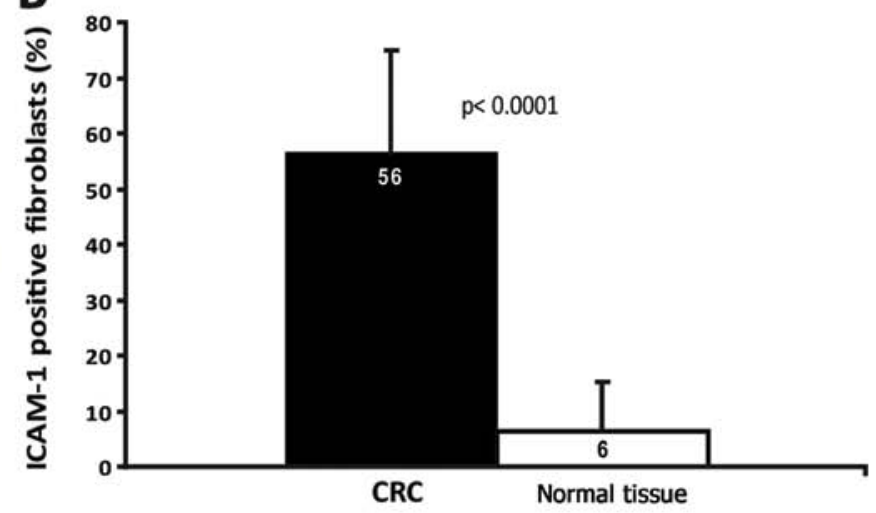

Figure 4. An increased number of ICAM-1-positive fibroblasts was present in the CRC tissue sections when compared to normal mucosa.(A) Immunohistochemical staining for ICAM-1 in paraffin-embedded tissue sections of CRC and normal mucosa. Arrows indicate positive staining. Magnification, x200. (B) Quantitative evaluation of ICAM-1-positive fibroblasts in CRC tissue and normal mucosa of all analyzed patients (p<0.001). ICAM-1, intercellular adhesion molecule-1; $\mathrm{CRC}$, colorectal cancer.

the tumor microenvironment. The so-called tumor-associated fibroblasts, fibroblasts activated by tumor-derived mediators such as transforming growth factor $\beta 1$ and platelet-derived growth factor, play a crucial role in these processes $(35,36)$.

Cellular adhesion molecules manage the interactions between cells and are therefore essential during the development and maintenance of the tissue architecture (15). ICAM-1 has received increased attention in cancer biology. Notably, membrane-bound ICAM-1 appears to protect tumor cells from separation from the tumor mass by tumor cell attachment to the extracellular matrix. Therefore, patients with increased membrane-bound ICAM-1 are found to have decreased lymph node and liver metastases as well as better differentiated tumors $(14,16,17)$.

In this report, we isolated and characterized tumor-associated-fibroblasts and compared them with corresponding normal tissue-derived fibroblasts. Both cell types were isolated from fresh CRC surgical specimens. Notably, TAFs presented with higher ICAM-1 expression when compared to the expression in NAFs, despite their separation from malignant cells during in vitro cultivation. These results were obtained by immunocytochemical staining of isolated fibroblast cultures for ICAM-1 and by performing adhesion assays with U937 cells to demonstrate the functional capacity of increased ICAM-1 expression. The performed tests revealed higher expression levels of ICAM-1 in 11 out of 12 CRC fibroblast pairs and a higher adhesion capacity of TAFs compared to corresponding NAFs in 8 out of 10 pairs. These results were reproducible after stimulation of cultures by IL- $1 \beta$ and subsequent ICAM-1 detection or with TNF- $\alpha$ and subsequent measurement of the cell adhesive capacity. In the paraffin-embedded tumor and mucosal tissue sections, we demonstrated a corresponding significant increase in the number of ICAM-1-positive fibroblasts in tumor tissue when compared to that in fibroblasts within the normal mucosa. This indicates, that i) the pro-inflammatory micromilieu of CRC leads to a higher expression of ICAM-1-positive cells in the tumor-surrounding tissue and that ii) these alterations are stable in fibroblasts when isolated from the surgical specimens and cultured in vitro.
These results support the theory that inflammation, as detected here by increased ICAM-1 expression in tumor-associated fibroblasts may i) enhance intratumoral adhesion of infiltrating antitumor immune cells and thereby foster a host reaction against the tumor. Moreover, fibroblasts stimulated by such an inflammatory reaction within the tumor do show stable expression of ICAM-1 in vitro and in vivo and ii) may stabilize the tumor by reducing tumor cell dissemination and consequently tumor progression may be reduced. As yet it is unclear how this reaction by TAFs influences the survival of CRC patients. Therefore, TAFs isolated from CRC will be an important tool for further elucidation of tumor microenvironment-associated processes in human CRC.

In conclusion, we demonstrated the successful isolation and cultivation of fibroblasts from fresh surgical specimens of patients with CRC. Importantly, the isolated cells preserved their commemoration which is affected by the microenvironment of the tumor. These fibroblasts are the main cellular component of the desmoplastic tumor stroma but their function in tumor progression is not well understood. Further elucidation of these cells and their interactions may open new options for non-surgical antitumor therapy.

\section{Acknowledgements}

We thank Christina von Kleinsorgen and Ingrid Mons for their technical support. The present study was supported by grants from 'Dr Robert Pfleger Stiftung' to V.S./E.N. and 'Deutsche Krebshilfe' (AZ 109510) to E.N./M.S., and IZKF-B20 to R.S.C./M.S./E.N.

\section{References}

1. Saunders $\mathrm{M}$ and Iveson T: Management of advanced colorectal cancer: state of the art. Br J Cancer 95: 131-138, 2006.

2. Parkin DM, Bray F, Ferlay J and Pisani P: Global cancer statistics, 2002. CA Cancer J Clin 55: 74-108, 2005.

3. Din FV, Theodoratou E, Farrington SM, Tenesa A, Barnetson RA Cetnarskyj R, et al: Effect of aspirin and NSAIDs on risk and survival from colorectal cancer. Gut 59: 1670-1679, 2010.

4. Wang D and Dubois RN: The role of COX-2 in intestinal inflammation and colorectal cancer. Oncogene 29: 781-788, 2010. 
5. Lakatos PL and Lakatos L: Risk for colorectal cancer in ulcerative colitis: changes, causes and management strategies. World J Gastroenterol 14: 3937-3947, 2008.

6. Strell C, Rundqvist H and Ostman A: Fibroblasts - a key host cell type in tumor initiation, progression, and metastasis. Ups J Med Sci 117: 187-195, 2012.

7. Benson V, McMahon AC and Lowe HC: ICAM-1 in acute myocardial infarction: a potential therapeutic target. Curr Mol Med 7: 219-227, 2007

8. Niessen HW, Lagrand WK, Visser CA, Meijer CJ and Hack CE: Upregulation of ICAM-1 on cardiomyocytes in jeopardized human myocardium during infarction. Cardiovasc Res 41 603-610, 1999.

9. Huang WC, Chan ST, Yang TL, Tzeng CC and Chen CC: Inhibition of ICAM-1 gene expression, monocyte adhesion and cancer cell invasion by targeting IKK complex: molecular and functional study of novel $\alpha$-methylene- $\gamma$-butyrolactone derivatives. Carcinogenesis 25: 1925-1934, 2004.

10. van de Stolpe A and van der Saag PT: Intercellular adhesion molecule-1. J Mol Med 74: 13-33, 1996.

11. Ksiazek K, Mikula-Pietrasik J, Catar R, Dworacki G, Winckiewicz M, Frydrychowicz M, et al: Oxidative stressdependent increase in ICAM-1 expression promotes adhesion of colorectal and pancreatic cancers to the senescent peritoneal mesothelium. Int J Cancer 127: 293-303, 2010.

12. Arteta B, Lasuen N, Lopategi A, Sveinbjörnsson B, Smedsrød B, and Vidal-Vanaclocha F: Colon carcinoma cell interaction with liver sinusoidal endothelium inhibits organ-specific antitumor immunity through interleukin-1-induced mannose receptor in mice. Hepatology 51: 2172-2182, 2010.

13. Alexiou D, Karayiannakis AJ, Syrigos KN,Zbar A, Kremmyda A Bramis I and Tsigris C: Serum levels of E-selectin, ICAM-1 and VCAM-1 in colorectal cancer patients: correlations with clinicopathological features, patient survival and tumour surgery. Eur J Cancer 37: 2392-2397, 2001.

14. Maeda K, Kang SM, Sawada T, Nishiguchi Y, Yashiro M, Ogawa Y, et al: Expression of intercellular adhesion molecule-1 and prognosis in colorectal cancer. Oncol Rep 9: 511-514, 2002.

15. Mantur M, Snarska J, Koper O, Dzieciol J, Plonski A and Lemancewicz D: Serum sICAM, sVCAM and sE-selectin levels in colorectal cancer patients. Folia Histochem Cytobiol 47: 621-625, 2009.

16. Taglia L, Matusiak D, Matkowskyj KA and Benya RV: Gastrinreleasing peptide mediates its morphogenic properties in human colon cancer by upregulating intracellular adhesion protein-1 (ICAM-1) via focal adhesion kinase. Am J Physiol Gastrointest Liver Physiol 292: G182-G190, 2007.

17. Wimmenauer S, Keller H, Rückauer KD, Rahner S, WolffVorbeck G, Kirste G, et al: Expression of CD44, ICAM-1 and N-CAM in colorectal cancer. Correlation with the tumor stage and the phenotypical characteristics of tumor-infiltrating lymphocytes. Anticancer Res 17: 2395-2400, 1997.

18. Kitagawa T, Matsumoto K and Iriyama K: Serum cell adhesion molecules in patients with colorectal cancer. Surg Today 28: 262-267, 1998 .

19. Sanchez-Rovira P, Jimenez E, Carracedo J, Barneto IC, Ramirez R and Aranda E: Serum levels of intercellular adhesion molecule 1 (ICAM-1) in patients with colorectal cancer: inhibitory effect on cytotoxicity. Eur J Cancer 34: 394-398, 1998.

20. Kang X, Wang F, Xie JD, Cao J and Xian PZ: Clinical evaluation of serum concentrations of intercellular adhesion molecule-1 in patients with colorectal cancer. World J Gastroenterol 11: $4250-4253,2005$.
21. Alexiou D, Karayiannakis AJ, Syrigos KN, Zbar A, Sekara E, Michail P, et al: Clinical significance of serum levels of E-selectin, intercellular adhesion molecule-1, and vascular cell adhesion molecule-1 in gastric cancer patients. Am J Gastroenterol 98: 478-485, 2003.

22. Nakata B, Hori T, Sunami T, Ogawa Y, Yashiro M, Maeda K, et al: Clinical significance of serum soluble intercellular adhesion molecule 1 in gastric cancer. Clin Cancer Res 6: 1175-1179, 2000.

23. Zhang GJ and Adachi I: Serum levels of soluble intercellular adhesion molecule-1 and E-selectin in metastatic breast carcinoma: Correlations with clinicopathological features and prognosis. Int J Oncol 14: 71-77, 1999.

24. O'Hanlon DM, Fitzsimons H, Lynch J, Tormey S, Malone C and Given HF: Soluble adhesion molecules (E-selectin, ICAM-1 and VCAM-1) in breast carcinoma. Eur J Cancer 38: 2252-2257, 2002.

25. Silva HC, Garcao F, Coutinho EC, De Oliveira CF and Regateiro FJ: Soluble VCAM-1 and E-selectin in breast cancer: relationship with staging and with the detection of circulating cancer cells. Neoplasma 53: 538-543, 2006.

26. Perabo F, Sharma S, Gierer R, Wirger A, Fimmers R, Steiner G, et al: Circulating intercellular adhesion molecule-1 (ICAM-1), vascular cell adhesion molecule-1 (VCAM-1) and E-selectin in urological malignancies. Indian J Cancer 38: 1-7, 2001.

27. Harning R, Mainolfi E, Bystryn JC, Henn M, Merluzzi VJ and Rothlein R: Serum levels of circulating intercellular adhesion molecule 1 in human malignant melanoma. Cancer Res 51: 5003-5005, 1991.

28. Grothey A, Heistermann P, Philippou S and Voigtmann R: Serum levels of soluble intercellular adhesion molecule-1 (ICAM-1, CD54) in patients with non-small-cell lung cancer: correlation with histological expression of ICAM-1 and tumour stage. Br J Cancer 77: 801-807, 1998.

29. Shimizu Y, Minemura M, Tsukishiro T, Kashii Y, Miyamoto M, Nishimori $\mathrm{H}$, et al: Serum concentration of intercellular adhesion molecule-1 in patients with hepatocellular carcinoma is a marker of the disease progression and prognosis. Hepatology 22: 525-531, 1995.

30. Christiansen I, Sundström C and Tötterman TH: Elevated serum levels of soluble vascular cell adhesion molecule-1 (sVCAM-1) closely reflect tumour burden in chronic B-lymphocytic leukaemia. Br J Haematol 103: 1129-1137, 1998.

31. Jackson AM, Alexandrov AB, Gribben SC, Esuvarnathan K and James K: Expression and shedding of ICAM-1 in bladder cancer and its immunotherapy. Int J Cancer 55: 921-925, 1993.

32. Budnik A, Grewe M, Gyufko K and Krutmann J: Analysis of the production of soluble ICAM-1 molecules by human cells. Exp Hematol 24: 352-359, 1996.

33. Franzke A, Probst-Kepper M, Buer J, Duensing S, Hoffmann R, Wittke F, et al: Elevated pretreatment serum levels of soluble vascular cell adhesion molecule 1 and lactate dehydrogenase as predictors of survival in cutaneous metastatic malignant melanoma. Br J Cancer 78: 40-45, 1998.

34. Schellerer VS, Croner RS, Weinländer K, Hohenberger W, Stürzl M and Naschberger E: Endothelial cells of human colorectal cancer and healthy colon reveal phenotypic differences in culture. Lab Invest 87: 1159-1170, 2007.

35. Desmoulière $A$, Guyot $C$ and Gabbiani G: The stroma reaction myofibroblast: a key player in the control of tumor cell behavior. Int J Dev Biol 48: 509-517, 2004.

36. Gonda TA, Varro A, Wang TC and Tycko B: Molecular biology of cancer-associated fibroblasts: can these cells be targeted in anti-cancer therapy? Semin Cell Dev Biol 21: 2-10, 2010. 